\title{
Retrospective Review of Interventional Radiology Treatment and Outcomes in Pediatric Liver Disease
}

\author{
Anna Purk ${ }^{1}$, Sean Pfaff ${ }^{3}$, Francis Marshalleck ${ }^{3}$, Jean Molleston ${ }^{2}$, \\ ${ }^{1}$ Indiana University School of Medicine, ${ }^{2}$ Riley Hospital for Children Department of \\ Gastroenterology, ${ }^{3}$ Riley Hospital for Children Department of Interventional Radiology
}

Background: Interventional radiology (IR) procedures offer less invasive and low risk options for the treatment of pediatric liver abnormalities, as well as life sustaining measures to bridge patients to transplant. Literature regarding IR interventions for pediatric liver disease is limited. Common conditions that can be treated with IR procedures include vascular anomalies: congenital portosystemic shunts, arteriovenous malformations (AVM), and Budd-Chiari malformation. Transjugular intrahepatic portosystemic shunt (TIPS) can be used to prevent recalcitrant variceal bleeding in portal HTN. Liver biopsy (percutaneous and transjugular) is used to sample the liver and can be targeted to tumors and other lesions. Percutaneous approaches to image biliary abnormalities and treat strictures are useful in children after liver transplant and also without.,

Objective: describe liver IR procedures and their outcomes in a single-center cohort study

Methods: Patients will be identified through retrospective chart review at Riley Hospital for Children to identify and record the diagnoses, interventions, and relevant outcomes for eligible patients. Descriptive statistics can then be performed. This data set can be used to help inform clinicians on indications and expected outcomes for relevant hepatic IR procedures.

Conclusion: It is anticipated that data collected for this project will show increased utilization of these procedures at the end of the study period compared to the beginning, and the complication rate for the IR procedure will be lower when compared to equivalent traditional procedures to treat the same condition. 